\title{
On Robust Two-Block Problems ${ }^{1}$
}

\author{
Babak Hassibi $^{2}$ and Thomas Kailath \\ Information Systems Laboratory \\ Stanford University, Stanford CA 94305
}

\begin{abstract}
In this paper we consider the following robust twoblock problem that arises in estimation and in fullinformation control: minimize the worst-case $H^{\infty}$ norm of a two-block transfer matrix whose elements contain $H^{\infty}$-norm-bounded modeling errors. We show that, when the underlying systems are single-input/singleoutput, and if the modeling errors are "small enough", then the robust two-block problem can be solved by solving a one-dimensional family of appropriatelyweighted "modeling-error-free" two-block problems. We also study the consequences of this result to a robust tracking problem, where the optimal solution can be explicitly found.
\end{abstract}

\section{Introduction}

In this paper we study the robust two-block problem

$$
\inf _{Q(\cdot) \in H^{\infty}} \sup _{\left(\Delta P_{1}(\cdot), \Delta P_{2}(\cdot)\right) \in B_{\delta}^{\infty}}\left\|T_{Q}\left(P_{1}+\delta P_{1}, P_{2}+\delta P_{1}\right)\right\|_{\infty},
$$

where

$$
\begin{gathered}
T_{Q}\left(P_{1}+\delta P_{1}, P_{2}+\delta P_{1}\right)= \\
{\left[\begin{array}{c}
P_{1}(z)+\Delta P_{1}(z)+\left(P_{2}(z)+\Delta P_{2}(z)\right) Q(z) \\
Q(z)
\end{array}\right]}
\end{gathered}
$$

and

$B_{\delta}^{\infty}=\left\{\left(\Delta P_{1}(\cdot), \Delta P_{2}(\cdot)\right),\left\|\left[\begin{array}{cc}\Delta P_{1}(\cdot) \quad \Delta P_{2}(\cdot)\end{array}\right]\right\|_{\infty} \leq \delta\right\}$, with $Q(\cdot), P_{1}(\cdot), P_{2}(\cdot), \Delta P_{1}(\cdot)$ and $\Delta P_{2}(\cdot)$ all scalar functions in $H^{\infty}$. This problem can be considered a robust version of the standard two-block problem

$$
\inf _{Q(\cdot) \in H_{\infty}}\left\|\left[\begin{array}{c}
P_{1}(z)+P_{2}(z) Q(z) \\
Q(z)
\end{array}\right]\right\|_{\infty},
$$

that arises in $H^{\infty}$ full-information control, and in $H^{\infty}$ estimation, which further allows one to consider possible modeling errors $\Delta P_{1}(\cdot)$ and $\Delta P_{2}(\cdot)$ for the nominal

\footnotetext{
${ }^{1}$ This work was supported in part by DARPA through the Department of Air Force under contract F49620-95-1-0525-P00001 and by the Joint Service Electronics Program at Stanford under contract DAAH04-94-G-0058-P00003.
}

plants $P_{1}(\cdot)$ and $P_{2}(\cdot)$. In problem (1) both the objective and the modeling errors are measured in the $H^{\infty}$ norm. Thus $\delta>0$ is a measure of the modeling error allowed for in (1).

\section{Main Result}

Problem (1) is, of course, a highly nonlinear problem and satisfactory solutions to date do not exist. In this paper, we show that for "small enough" modeling errors problem (1) can be solved by doing a one-dimensional search over the solution of a certain family of weighted standard two-block problems.

Thus, consider the solution to the following weighted two-block problem:

$$
\begin{aligned}
f(\epsilon) \triangleq \inf _{Q(\cdot) \in H^{\infty}}\left\|\left[\begin{array}{c}
\sqrt{1+\epsilon}\left(P_{1}(z)+P_{2}(z) Q(z)\right) \\
\sqrt{1+\left(1+\frac{1}{\epsilon}\right) \delta^{2}} Q(z)
\end{array}\right]\right\|_{\infty}^{2} \\
+\left(1+\frac{1}{\epsilon}\right) \delta^{2}, \quad \epsilon>0 .
\end{aligned}
$$

The above problem can be readily solved for any value of $\epsilon$ (say, by using Riccati-based techniques when $P_{1}(\cdot)$ and $P_{2}(\cdot)$ are rational), and so $f(\epsilon)$ is easy to compute. Moreover, it can be shown that $f(\cdot)$ is, in general, a nonconvex continuous function of $\epsilon$. Suppose now that we perform a one-dimensional search over $\epsilon>0$, and determine

$$
\epsilon^{*} \triangleq \inf _{\epsilon>0} f(\epsilon)
$$

Then we have the following result.

\section{Theorem 1 (Robust Two-Block Problem)}

There exists a $\bar{\delta}>0$, such that for all $\delta<\bar{\delta}$, the solution to the robust two-block problem (1) can be found from the solution to the weighted two-block problem

$$
\inf _{Q(\cdot) \in H^{\infty}}\left\|\left[\begin{array}{c}
\sqrt{1+\epsilon^{*}}\left(P_{1}(z)+P_{2}(z) Q(z)\right) \\
\sqrt{1+\left(1+\frac{1}{\epsilon^{*}}\right) \delta^{2}} Q(z)
\end{array}\right]\right\|_{\infty}^{2}+\left(1+\frac{1}{\epsilon^{*}}\right) \delta^{2} .
$$


The above theorem states that if the modeling error is less than $\bar{\delta}>0$, then the robust two-block problem (1) can be solved using a one-dimensional search over a family of weighted two-block problems. Moreover, the solution to (1) is the same as the solution to a certain weighted two-block problem, with optimal weighting determined by $\epsilon^{*}$. This has rather interesting physical implications since it states that the modeling errors $\Delta P_{1}(\cdot)$ and $\Delta P_{2}(\cdot)$ can be dealt with by appropriately weighting the modeling-error-free two-block problem (2).

Of course, this result raises several issues:

- How does Theorem 1 generalize to matrix plants?

- How does Theorem 1 generalize to four-block problems?

- How large is the value of $\bar{\delta}$ in Theorem 1 ?

Currently all three questions are open. To gain some insight into the third question, let us consider the robust tracking problem.

\section{Robust Tracking}

The $H^{\infty}$ tracking problem corresponds to $P_{1}(z)=1$ and $P_{2}(z)=-P(z)$, so that the robust tracking problem takes the form

$\inf _{Q(\cdot) \in H^{\infty}} \sup _{\|\Delta P(\cdot)\|_{\infty} \leq \delta}\left\|\left[\begin{array}{c}1-(P(z)+\Delta P(z)) Q(z) \\ Q(z)\end{array}\right]\right\|_{\infty}$

The modeling-error-free tracking problem,

$$
\inf _{Q(\cdot) \in H^{\infty}}\left\|\left[\begin{array}{c}
1-P(z) Q(z) \\
Q(z)
\end{array}\right]\right\|_{\infty} \triangleq \gamma_{o p t},
$$

has been studied in [1], where it is shown:

- If $P(z)$ is minimum phase, then

$$
\gamma_{o p t}=\frac{1}{1+\min _{\omega \in[0,2 \pi]}\left|P\left(e^{j \omega}\right)\right|^{2}} .
$$

- If $P(z)$ is minimum phase, then

$$
\gamma_{o p t}=1 \text {. }
$$

Let us now return to problem (6). Clearly, if $P(z)$ is nonminimum phase, we can always obtain an $H^{\infty}$ norm of unity in the objective cost by setting $Q(\cdot)=0$. This is the same value obtained in the modeling-error-free case. Therefore, let us focus on the case where $P(z)$ is minimum phase.

Here we will have to distinguish between two cases: (i) $\delta \geq \min _{\omega \in[0,2 \pi]}\left|P\left(e^{j \omega}\right)\right|^{2} \triangleq p_{\min }$. In this casc, there exist modeling errors for which $P(\cdot)+\Delta P(\cdot)$ is non-minimum phase. Thus here the best choice is $Q(\cdot)=0$, which results in an $H^{\infty}$ norm of unity.

(ii) $\delta<\min _{\omega \in[0,2 \pi]}\left|P\left(e^{j \omega}\right)\right|^{2} \triangleq p_{m i n}$. In this case, $P(\cdot)+\Delta P(\cdot)$ is always minimum phase.

Thus, clearly the case of interest is case (ii), above. The next result shows that for this case, the optimallyweighted two-block problem aluays solves (6).

Theorem 2 (Robust Tracking) Consider problem (6) and suppose that

$$
\delta<\min _{\omega \in[0,2 \pi]}\left|P\left(e^{j \omega}\right)\right|^{2} \triangleq p_{\min }
$$

Then the solution to problem (6) is given by the solution to the problem,

$$
\inf _{Q(\cdot) \in H^{\infty}}\left\|\left[\begin{array}{c}
\sqrt{1+\epsilon^{*}}(1-P(z) Q(z)) \\
\sqrt{1+\left(1+\frac{1}{\epsilon^{*}}\right) \delta^{2}} Q(z)
\end{array}\right]\right\|_{\infty}^{2}
$$

where

$$
\epsilon^{*}=\arg \min _{\epsilon>0} \inf _{Q(\cdot) \in H^{\infty}}\left\|\left[\begin{array}{c}
\sqrt{1+\epsilon}(1-P(z) Q(z)) \\
\sqrt{1+\left(1+\frac{1}{\epsilon}\right) \delta^{2}} Q(z)
\end{array}\right]\right\|_{\infty}^{2} .
$$

In particular, when $p_{\min }<2$, we have

$$
\epsilon^{*}=\frac{\delta\left(p_{\min }-\delta\right)}{1-\delta\left(p_{\min }-\delta\right)},
$$

and the optimal $H^{\infty}$ norm becomes

$$
\gamma_{o p t}=\frac{1}{1+\left(p_{\min }-\delta\right)^{2}},
$$

which is the same as that obtained from max-min problem:

$$
\sup _{\|\Delta P(\cdot)\|_{\infty} \leq \delta} \inf _{Q(\cdot) \in H^{\infty}}\left\|\left[\begin{array}{c}
1-(P(z)+\Delta P(z)) Q(z) \\
Q(z)
\end{array}\right]\right\|_{\infty} .
$$

Thus the method presented here for solving robust twoblock problems always works for the tracking problem. Moreover, it is interesting that for $p_{\min }<2$ the solutions to the min-max problem (6) and the max-min problem (14) coincide. [In general we have min-max $\geq$ $\max -\min$.

\section{References}

[1] B. Hassibi and T. Kailath. Tracking with an $H^{\infty}$ criterion. In Proceedings of the 36th IEEE Conference on Decision and Control, San Diego, CA, Dec. 1997. 